\title{
Comparative study between Tramadol and Midazolam as an admixture to bupivacaine inUltrasound guided supraclavicular brachial plexus block.
}

\author{
MostafaM .Abdelzaher(Resident);Hamza Abo-alam Mahmoud(M.D);Ahmed Mohamed A. \\ Abdel-Maboud(M .D);Khaled M ohamed Hassaan(M .D).
}

Department of Anesthesiology, Sohag University, Egypt.

\begin{abstract} recorded in both groups using pain VAS score.

\section{Introduction}

Regional anesthesia for upper limb surgery offers significant benefits over general anesthesia (1). These benefits include superior intra-operative pain control, attenuation of the surgical stress response, minimal systemic impairment, lower incidence of postoperative nausea and vomiting, excellent localized postoperative analgesia, and decrease hospital discharge time and cost (2).
\end{abstract}

Background:This is a prospective, randomized, double blind study to evaluate the postoperative analgesia following ultrasound guided supraclavicular brachial plexus block with Tramadol or Midazolam as an admixture to bupivacaine in upper extremity surgery.

Patientsand methods:Total 60 patients of ASA I and II undergoing upper extremity surgery under ultrasound guided brachial plexus block with Bupivacaine were randomly divided in to two groups; one group received Tramadol $(1 \mathrm{mg} / \mathrm{kg})$ and the other group received midazolam $(0.05 \mathrm{mg} / \mathrm{kg})$ as an admixture to Bupivacaine. The duration of postoperative analgesia was

Results: The mean duration of postoperative analgesia in the Midazolam group was $650 \pm 47.49$ minutes while in the tramadol group it was $508 \pm 30.44$ minutes.

Conclusion: We concluded that midazolamwith local anaesthetic prolongs postoperative analgesiasignificantly than Tramadol $(\mathrm{P}<0.0001)$ when used as admixture to local anaesthetic inUltrasound guided brachialplexus block in upper extremity surgery.

Key words: Analgesia, Brachial plexus, Ultrasound, Midazolam, Tramadol.

With increasing emphasis on patient safety and better patient outcomes, Ultrasound guided regional anesthesia is becoming more popular. As it provides clinicians with a real time image useful for visualizing anatomical structures, needle placement and local anesthetic spread .Supraclavicular brachial plexus block provides consistently effective anesthesia to the upper extremity (3).

Several adjuvants have been studied so far including, clonidine, tramadol, midazolam, neostigmine, bicarbonate, and dexamethasone .Addition of any of the above agents to brachial plexus block is supposed to prolong the analgesic effect without any unwanted systemic effects and also reduce the total requirement of local anesthetic used (4).

Midazolam, a water soluble benzodiazepine, is known to produce antinociception and enhance the effect of local anesthetics. Tramadol hydrochloride is a synthetic opioid with a relatively weak affinity to opioid receptors, it inhibits the reuptake of Nor-epinephrineand serotonin from the nerve endings (5) .The effect of Tramadol as an adjuvant to local anesthetics in supraclavicular brachial plexus block is to enhance the onset time and duration of both sensory and motor block, and post-operative analgesia were observed (6).

\section{Patients And Methods}

Main objective of this study was to determine whetherBupivacaine in combination with Tramadol orMidazolam 
increases the duration of analgesiafollowing ultrasound guided supraclavicular brachial plexus block in upperextremity surgery. This is a prospective, randomized, double blind study of60 patients (30 patients in each group) of AmericanSociety of Anaesthesiologists (ASA) physical grade III,aged between 16- 65 years, undergoing upper extremitysurgery (elbow, forearm and hand) under supraclavicular brachial plexus block, Approval for the study wastaken from KMC ethical committee and written; informedconsent was taken from the patients.

Exclusion criteria were Patients with a history of significant neurological, psychiatric, neuromuscular, cardiovascular, severe pulmonary, renal, hepatic disease. Alcoholism or drug abuse, Pregnancy or lactating women, Patients receiving chronic analgesic therapy or current opioid use, Morbid obesity, diabetes, peripheral vascular disease, Known allergy to study medications, Having infection or previous surgery in the block site, Patients with peripheral neuropathy, patient with Coagulopathy, Patient's refusal of the study protocol.

The patients were randomly allocated to two groups, 30 patients each: Group $\mathrm{T}$ (Tramadol group):Patient received $30 \mathrm{ml}$ of $0.375 \%$ bupivacaine plus $1 \mathrm{mg} / \mathrm{kg}$ tramadol in $2 \mathrm{ml}$ saline, Group $\mathrm{M}$ (midazolam group):Patient received $30 \mathrm{ml}$ of 0.375 bupivacaine plus $50 \mathrm{mic} / \mathrm{kgMidazolam}$ in $2 \mathrm{ml}$

After arrival to the anesthetic room, the peripheral intravenous (IV) line was placed in the non-operative upper limb and an infusion started with normal saline. Supplemental oxygen (3L/minute, was delivered by nasal cannula and routine anesthesia monitoring, including noninvasive arterial blood pressure, heart rate, and pulse oximetry were applied, The skin of the supraclavicular region was disinfected with a $10 \%$ povidone-iodine solution we use a SonoScape ultrasound machine, with a linear high frequency (8-13 $\mathrm{MHz}$ ) under complete aseptic conditions,
The block was performed with the patient in supine position with the head turned away from the side to be blocked. The arm is adducted closely. Adherence to strict anatomic landmarks is of lesser importance for the ultrasound-guided supraclavicular block than for the surface anatomy techniques. However, knowledge of the underlying anatomy and the position of the brachial plexus in relation to the subclavian artery, first rib, and pleura are very important for the success and safety of the technique.Scanning is usually started just above the clavicle at approximately its midpoint.

The transducer is positioned in the transverse plane immediately superior to the clavicle at approximately its midpoint. The transducer is tilted caudally to obtain a cross-sectional view of the subclavian artery. The brachial plexus is seen as a collection of hypoechoic oval structures lateral and superficial to the artery.

Using a 25- to 27-gauge needle, 1 to $2 \mathrm{~mL}$ of local anesthetic is injected into the skin 1 $\mathrm{cm}$ lateral to the transducer to decrease the discomfort during needle insertion. Local infiltration may not be necessary in well premedicated patients. The needle should never be inserted deeper than $1 \mathrm{~cm}$ to avoid inadvertent puncture of and injection into the brachial plexus. Always observe the distribution of the local anesthetic during administration by injecting small amounts of the local anesthetic as the needle advances through tissue layers (hydro-localization). The block needle is then inserted in-plane toward the brachial plexus

The needle is inserted on the lateral side of the probe since the plexus is located lateral to the subclavian artery. The entrance point is $1 \mathrm{~cm}$ away from the probe to decrease the angle of insertion, and it is moved from lateral to medial. The needle is placed in the long axis, in plane to the probe, and should be visualized at all times. This is especially important for this block since the needle can cause a pneumothorax if not visualized at all times. 
To confirm lung parenchyma, ask the patient to take a deep breath; the characteristic multiple parallel lines of the pleura layers move, and the change in lung appearance occurs. The needle is advanced under direct visualization toward the angle formed by the first rib and the subclavian. A small amount of local anesthetic solution is injected to confirm the location of the needle tip; this can be done while slowly advancing the needle.

The injected volume gently expands the connective tissue surrounding the nerves, which is called hydro dissection. This allows a clear path for the needle, decreasing the chance of nerve damage of the nerves by the needle. We should see the spread of local anesthetic in the angle between the first rib and the subclavian artery. Color Doppler can be done to confirm that the artery is visualized if any doubt exists. The subclavian vein is medial and superficial to the artery and is compressible. We should aspirate every 3-5 $\mathrm{ml}$ during injection to prevent vascular injection. Keep the nerve bundle centered in the image. If the needle is difficult to visualize, rock the probe back and forth until the needle is visualized.

The following parameters were assisted after institution of the blockade:Onset and duration of sensory block. Onset and duration of motor block.Duration of postoperative analgesia and total consumption of analgesics.Side effect and complication if present

Sensory blockade was tested using pin prick method along the distribution of the four nerves (median nerve, radial nerve, ulnar nerve and Musculocutaneous nerve) compared with contra lateral limb as a reference, $0=$ normal sensation, $1=$ loss of sensation of pinprick, $2=$ loss of sensation of touch.
Onset of sensory blockade is considered as the time interval between the end of local anesthetic administration and loss of sensation to pin prick.

The duration of analgesia is defined as the time interval between the ends of local anesthetic administration to the time when patient had VAS (Visual analogue scale) score of $\geq 4$.Patient will receive analgesia in the form of pethidine $0.5 \mathrm{mg} / \mathrm{kg}$ per dose

Motor blockade assessment was done using the Modified Bromage scale for upper extremities on a three point scale. Grade $0=$ normal motor function with full flexion/extension of elbow, wrist and fingers. Grade $1=$ decreased motor strength with ability to move fingers and/or wrist only.Grade $2=$ complete motor blockade with inability to move fingers.

Onset of motor blockade is considered as the time interval between the end of local anesthetic administration and inability to move fingers. The duration of motor blockade is defined as the time interval between the end of local anesthetic administration and the recovery of complete and motor function of the hand.

The surgery was allowed to proceed when complete anesthesia was achieved. Intra operatively HR, NIBP, SPO2 were monitored every 5 minute.Post operatively, motor blockade and VAS score were assessed at 4, 8, 16, 18, 19, 20, 21, 22, 23, 24hr.The first request for analgesics and total pethidine dose was documented.

Sedation level will be assessed by Modified Ramsay score; $0=$ Irritable, $1=$ Awake, $2=$ Lightly sedated, $3=$ Moderately sedated , follows simple commands, 4=Deeply sedated responds to non-painful stimuli, $5=$ Deeply sedated, responds only to painful stimuli, 6=Deeply sedated , unresponsive to painful stimuli.

\section{RESULTS}

The minimum age of the patient was 16 years and the maximum age was 60 years. The mean age of the patients in midazolam group was $37.73 \pm 15.63$ and in tramadol group were $35.27 \pm 15.31$ years. Age distribution between two groups is similar with no significant difference, $\mathrm{P}$ value $>0.05$, also no difference in the other demographic data, 
The mean time for onset of sensory block in midazolam group was $22.73 \pm 1.68$ min and in tramadol group was $23.1 \pm 1.73 \mathrm{~min}$. The statistical analysis showed that, the time for onset of sensory block in both groups was non-significant ( $p>0.05$ ). The mean time for onset of motor block in midazolam group was $45.2 \pm 2.96 \mathrm{~min}$ and in tramadol group was $46.5 \pm 2.97 \mathrm{~min}$. The statistical analysis showed that, the time for onset of motor block was non-significant ( $>>0.05)$. Patients of both groups were observed for 24 hours. Time was noted when the patient asked for rescue analgesics. The mean duration of sensory block in midazolam group was $510 \pm 30.51 \mathrm{~min}$ and in tramadol group was $498 \pm 32.10 \mathrm{~min}$. The statistical showed that the duration of sensory block in both groups was non-significant ( $p>0.05$ ). The mean duration of motor block in midazolam group was $416 \pm 49.66 \mathrm{~min}$ and that of tramadol group was $392 \pm 68.20 \mathrm{~min}$. The statistical analysis showed non-significant difference between two groups, with $\mathrm{p}$ value $>0.05$.

The mean duration of post-operative analgesia in midazolam group was $650 \pm 47.49$ min but in tramadol group was $508 \pm 30.44 \mathrm{~min}$. the statistical analysis showed highly significant difference between two groups $\mathrm{P}$ value $<0.0001$, table (1).

Table (1) Comparison between midazolam and tramadol group as regard onset and duration.

\begin{tabular}{|c|c|c|c|}
\hline Variables & Midazolam & Tramadol & P value \\
\hline Onset of sensory block & $22.73 \pm 1.68$ & $23.1 \pm 1.73$ & 0.41 \\
\hline Onset of motor block & $45.2 \pm 2.96$ & $46.5 \pm 2.97$ & 0.10 \\
\hline Duration of surgery & $126.04 \pm 53.08$ & $116 \pm 47.09$ & 0.48 \\
\hline Duration of analgesia & $\mathbf{6 5 0} \pm 47.49^{*}$ & $\mathbf{5 0 8} \pm 30.44$ & $<0.0001$ \\
\hline Duration of sensory block & $\mathbf{5 1 0} \pm 30.51$ & $\mathbf{4 9 8} \pm 32.10$ & 0.14 \\
\hline Duration of motor block & $\mathbf{4 1 6} \pm 49.66$ & $\mathbf{3 9 2} \pm \mathbf{6 8 . 2 0}$ & $\mathbf{0 . 1 2}$ \\
\hline
\end{tabular}

Data presented as mean $\pm S D$ (standard deviation).

$\wedge$ Significant difference $P$ value $<0.05$.

* Highly significant difference, $P$ value $<0.0001$.

The mean of total dose of consumed pethidine in 24 hours which was $62.33 \pm 18.32$ in midazolam group and $93.33 \pm 18.82$ in tramadol group with highly significant difference between two groups, $\mathrm{P}$ value $<0.0001$.

In tramadol group, all patients were awake and alert and had sedation score of (1) according to Modified Ramsay Sedation Scale, meanwhile In midazolam group, sedation corresponding to score 2 was observed in some patients between $20 \mathrm{~min}$ from time of injection and $60 \mathrm{~min} .27 \%$ of patients at $20 \mathrm{~min}, 53 \%$ of patients at $30 \mathrm{~min}$ and $20 \%$ of patients at $60 \mathrm{~min}$ had sedation score of (2). None of the patients had sedation score of 3 and above during the study period. Statistical analysis of sedation score by chi-square test showed that the difference in sedation score was highly significant $P$ value $<0.0001$,

In the first 4 hrs. Postoperative the VAS was similar in both groups and it increased steadily in both groups with time. However, the VAS was always less in midazolam group compared to that of tramadol group, with significant to highly significant differences, $\mathrm{p}$ value $<0.0001$.

MAP ranged from $77.06 \pm 12.63$ to $98.57 \pm 6.18 \mathrm{~mm}$ of $\mathrm{Hg}$ in midazolam group and ranged from $72.73 \pm 7.82$ to $99.8 \pm 8.41 \mathrm{~mm} \mathrm{Hg}$ in tramadol group. The statistical analysis showed that there was no significant difference in mean blood pressure between two groups $\mathrm{p}$ value $>0.05$ 
The mean pulse rate which ranged from $68.33 \pm 2.95 \mathrm{~b} / \mathrm{m}$ to $79.63 \pm 4.48 \mathrm{~b} / \mathrm{m}$ in midazolam group, and from $67.33 \pm 1.94 \mathrm{~b} / \mathrm{m}$ to $79.03 \pm 3.48 \mathrm{~b} / \mathrm{m}$ in tramadol group with no significant difference between two groups, $\mathrm{P}$ value $>0.005$.

The mean $\mathrm{O} 2$ saturation ranged from $98.5 \pm 1.33 \%$ to $99.23 \pm 0.97 \%$ in midazolam group, the mean $\mathrm{O} 2$ saturation ranged from $97.67 \pm 1.54 \%$ to $99.6 \pm 0.93 \%$ in tramadol group. The statistical showed that there was no significant difference in $\mathrm{O} 2$ saturation between the two groups $(\mathrm{p}>$ 0.05 .

The respiratory rate in concerned groups which ranged from $14.23 \pm 1.55 \mathrm{c} / \mathrm{m}$ to $15.77 \pm 1.77 \mathrm{c} / \mathrm{m}$ in midazolam group, and ranged from $13.53 \pm 1.80 \mathrm{c} / \mathrm{m}$ to $16.2 \pm 1.52 \mathrm{c} / \mathrm{m}$ in tramadol group with no significant difference between two groups $\mathrm{P}$ value $>0.05$

In midazolam group no unwanted side effects have been occurred; unlike in tramadol group nausea was recorded in two patients and vomiting in one patient among 30 patients of tramadol group

\section{DISCUSSION}

Brachial plexus block is a popular and widely employed regional block technique for peri operative anesthesia and analgesia for surgeries of the upper extremity. Regional nerve block avoids the unwanted effects of the anesthetic drugs used during general anesthesia and the stress of laryngoscopy and tracheal intubation. The supraclavicular block is often quicker to perform and may result in fewer side effects than the interscalene block. Compared to the infra clavicular block and axillary blocks, the successful achievement of adequate anesthesia for surgery of the upper extremity is about the same with supraclavicular block. Unlike the interscalene block - which results in diaphragmatic hemiparesis in all subjects - only half of those who undergo supraclavicular block experience this side effect. Disadvantages of the supraclavicular block include the risk of pneumothorax, which is estimated to be between 1-4\% when using paresthesia (blind technique) or peripheral nerve stimulator guided techniques which can be avoided by ultrasound guidance (7)

Ultrasound guidance had improved the success and decreased the complications rate in regional anesthesia in general. The use of two-dimensional ultrasonic imaging to localize the brachial plexus has been highly successful in several approaches. Modern ultrasound machines are capable of imaging individual roots to their cords in the supraclavicular region.
The sonographic image can be used to guide the injection needle while minimizing the risk of injury of adjacent structures. Local anesthetics alone provide analgesia for not more than $4-8 \mathrm{hr}$.Increasing the duration of local anesthetic action is often desirable because it prolongs surgical anesthesia and analgesia. Different additives had been used to prolong regional blockade as dexamethasone, opioid, ketamine, midazolam...etc. but the results were inconclusive or associated with side effects (8). The aim of our study was to compare the effect of midazolam $(50 \mathrm{mic} / \mathrm{kg})$ and tramadol $(1 \mathrm{mg} / \mathrm{kg})$ when added to bupivacaine in ultrasound guided supraclavicular brachial plexus block for upper limb surgeries as regard to time of onset of sensory and motor blocks, duration of post-operative analgesia, motor block duration, hemodynamic variability and side effects as nausea, vomiting, hypotension, bradycardia, hematoma, pneumothorax and local anesthetics toxicity if present.

In our study, we found that addition of midazolam to bupivacaine results in significant prolongation of duration of postoperative analgesia, lower analgesic consumption and lower incidence of adverse effects compared to tramadol.

Patient demographic data showed that the majority of cases were males and there is no significant difference between the two groups regarding gender of the patients. The mean age of the study groups was around 37 
years in midazolam group and 35 years in tramadol group, with no significant difference, that can be explained by that trauma and accidents are more common among active young age more than old aged people. The weight of the study group was slightly higher in midazolam group compared to the tramadol group, with no significant difference.

In our study, the onset of sensory and motor block, the duration of sensory and motor block were similar in both groups, with no significant difference between two groups, $\mathrm{p}$ value $>0.05$. Meanwhile, the analgesia duration was significantly higher among midazolam group $(650 \pm 47.49)$ compared to tramadol group $(508 \pm 30.44) \mathrm{p}$ value $<0.001$ This comes in agreement with a study done by Anil Kumar et al., 2016. In midazolam group they found that the mean duration of analgesia in midazolam group was $13.65 \pm$ $2.01 \mathrm{hrs}$. In our study in midazolam group it was $10.8 \pm 0.78 \mathrm{hrs}$, it is nearly similar. The mean duration of motor block in midazolam group in Anil Kumar 2016 study and our study were $7.23 \pm 1.01 \mathrm{hrs} 6.9 \pm 0.83 \mathrm{hrs}$ respectively with no significant difference. Mean time of onset of sensory and motor blocks was earlier in midazolam group 9.52 \pm 1.37 and $12.3 \pm 1.35$ respectively compared to the midazolam group in our study which were $22.73 \pm 1.68$ and $45.2 \pm 2.96$ respectively as they used inside-sheath technique while we used U/S guided outside-sheath technique. They concluded that addition of midazolam to local anesthetic drugs in supraclavicular brachial plexus block significantly prolongs the duration of analgesia and duration of motor block in patients undergoing upper limb surgeries.

Our study results come in contact with a study done by Nasreen, et al 2007. They found that in midazolam group the onset of sensory block was $14 \pm 3.1 \mathrm{~min}$, the onset of motor bock was $10.5 \pm 2.40 \mathrm{~min}$, in our study in midazolam group the onset of sensory block was $22.73 \pm 1.68 \mathrm{~min}$, the onset of motor bock was $45.2 \pm 2.96$ min. We can notice that there is significant difference in the onset of block that can be explained by that they used inside-sheath technique unlike us, we used U/S guided outside-sheath technique.

In Nasreen 2017 study the duration of sensory block was $9.30 \pm 4.5 \mathrm{hrs}$ and the duration of motor block was 7.6 $\pm 3.2 \mathrm{hrs}$. In our study the duration of sensory block was $8.5 \pm 0.5 \mathrm{hrs}$ and the duration of motor block was $6.9 \pm 0.68 \mathrm{hrs}$, with no significant difference between two studies.

In Nasreen 2017 rescue analgesic in midazolam group was $60.23 \pm 47 \mathrm{mg}$ similar to ours $62.33 \pm 18.32 \mathrm{mg}$ pethidine, Also Peri-operatively sedation scores in midazolam group, 6 patients at 10 minutes, 10 patients at 20 minutes and 15 patients at 30 minutes were sedated and responded to verbal stimulation (score 2). The highest sedation score was 2 and no patient had sedation score of 3 or more that, in our study In midazolam group, sedation corresponding to score 2 was observed in some patients between $20 \mathrm{~min}$ from time of injection and 60 min. 8 patients at $20 \mathrm{~min}, 16$ of patients at $30 \mathrm{~min}$ and 6 of patients at $60 \mathrm{~min}$ had sedation score of (2). None of the patients had sedation score of 3 or above during the study period.

Our results were supported by a study done by Shrestha et al., 2007 In tramadol group the authors found that the duration of analgesia was $8.16 \pm 0.48$ hours; in our study in tramadol group postoperative analgesia was $8.5 \pm 0.51 \mathrm{hrs}$ with no significant difference between Shrestha study and ours as regard to results of tramadol group.

Our study results come in agreement with a study done by Abrar Hussein; et al 2015, in tramadol group, the onset of sensory block $16.20 \pm 0.96 \mathrm{~min}$ and the onset of motor block was $22.83 \pm 8.1 \mathrm{~min}$, in our study in midazolam group the onset of sensory block was $22.73 \pm 1.68 \mathrm{~min}$, the onset of motor bock was $45.2 \pm 2.96 \mathrm{~min}$. We can notice that there is significant difference in the onset of block that can be explained by that they used inside-sheath technique unlike us; we used U/S guided outside-sheath technique. The duration of sensory block was 
$8.3 \pm 0.54$ hours; the duration of motor block was $8.3 \pm 0.54$ hours, in our study the duration of sensory block was $8.5 \pm 0.5 \mathrm{hrs}$. And the duration of motor block was $6.9 \pm 0.68 \mathrm{hrs}$. With no significant difference between two studies. First request for rescue analgesia was $8.1 \pm 0.71$ hours; it is similar to ours as First request for rescue analgesia was $8.5 \pm 0.51 \mathrm{hrs}$.Abrar Hussein 2015 study in midazolam group showed no complications like us.

Our results comes in contradict with Ahmet Can Senel et al., 2014 who designed a study to assess the effect of tramadol and ketamine, 50mg, added to ropivacaine in brachial plexus anesthesia.. 60 patient ASA physical statuses I and II patients, between 18 and 60 years of age, scheduled for forearm and hand surgery under axillary brachial plexus block, were allocated to 3 groups (20 patients for each). Group $\mathrm{R}$ received $0.375 \%$ ropivacaine in $40 \mathrm{~mL}$, group RT received $0.375 \%$ ropivacaine in $40 \mathrm{~mL}$ with $50 \mathrm{mg}$ tramadol, and group RK received $0.375 \%$ ropivacaine in $40 \mathrm{~mL}$ with $50 \mathrm{mg}$ ketamine for axillar brachial plexus block. The time of onsets and the duration of sensory and motor blocks, duration of analgesia, hemodynamic parameters, and adverse events (nausea, vomiting, and feeling uncomfortable) were recorded.

In tramadol group They found that the time of onset of sensory block was $8.17 \pm 0.33$ min, The time of onset of motor block was $10.8 \pm 0.38 \mathrm{~min}$, the duration of sensory block was $13.16 \pm 0.40 \mathrm{hrs}$, the duration of motor block was $15.8 \pm 041 \mathrm{hrs}$ and the duration of analgesia was significantly long it was $24.90 \pm 0.33 \mathrm{hrs}$, Two patients were considered sedated in tramadol group (score

\section{REFERENCES}

1. AnandLK,JindalR(2009). Regional anaesthesia for upper extremity in orthopaedics

:areviewarticle.PunjabJ,Orthopaedics 11:1-8.

2. GebhardRE,Al-

SamsamT,GregerJ,etal.Distal nerve block at the wrist for outpatient carpal
2). The difference between our study and Ahmet Can Senel can't be neglected as in our study in tramadol group we found that the time of onset of sensory block was $23.1 \pm 1.73 \mathrm{~min}$, The time of onset of motor block was $46.5 \pm 2.97 \mathrm{~min}$, this difference can explained by their usage of ropivacaine as a local anesthetic instead of bupivacaine as ropivacaine is characterized by rapid onset of action and longer duration of block than bupivacaine, also they used the inside-sheath technique. The duration of sensory block was $8.3 \pm 0.54$ hours, the duration of motor block was $6.5 \pm 1.1$ hours and the duration of analgesia was $8.5 \pm 0.51$ hours.

We can notice significant difference in duration of sensory and motor block and duration of post-operative analgesia in that study which may be explained by use of ropivacaine not bupivacaine, ropivacaine is characterized by rapid onset of action and longer duration of block and analgesia than bupivacaine.

No sedated cases were recorded in our study in tramadol group, meanwhile in midazolam group sedation corresponding to score 2 was observed in some patients between $20 \mathrm{~min}$ from time of injection and $60 \mathrm{~min} .8$ patients at $20 \mathrm{~min}, 16$ patients at $30 \mathrm{~min}$ and 6 patients at $60 \mathrm{~min}$ had sedation score of (2).

\section{CONCLUSION}

The addition of midazolam to bupivacaine resulted in significant prolongation of duration of post-operative analgesia, lower analgesic consumption and lower incidence of complications.

The use of ultrasound helped us in good localization of brachial plexus and prevented occurrence of major complications

tunnel surgery offer intraoperative cardiovascular stability and reduce discharge time.AnesthAnalg 2002;95:351.

3. Vermeylenk,Engelens,SermusL,Soeten sF,Van de veldeM.supraclavicular brachial plexus blocks;review and 
current practice .ActaAnaesthesiolBelg 2012,63;15-21.

4. Duma A, Urbanek B, Sitzwohl C, Kreiger A, Zimpfer M, Kapral S. Clonidine as an adjuvant to local anaesthetic axillary brachial plexus block: A randomized, controlled study. Br J Anaesth. 2005;94:112-6.

5. RobauxS.,Blunt C.,VielF.e, Cuvillon P., Girad F. and Bouaziz H.(2004) Tramadol added to bupivacaine for axillary brachial plexus block improve postoperative analgesia.
6. Gear, R.W., Miaskowski, C. and Gorden, N.C. (1999) The The Mu receptors tramadol Produces Gender and Dose DependentAnalgesia and Antianalgesia in Patients with Postoperative Pain. Pain, 83, 339-345.

7. De Tran $\mathrm{QH}$, Clemente A, Doan J, Finlayson RJ (2007). (8): 54. Canadian Journal of Anesthesia. review of approaches and techniques"662-74.

8. wakhlo, Woolf CJ: Recent advances in the pathophysiology of acute pain. $\mathrm{Br} \mathrm{J}$ Anaesth 2006; 63:139-146. 